\title{
Current Treatment Patterns of Aplastic Anemia in China: A Prospective Cohort Registry Study
}

\author{
Xiao-Fan Zhu ${ }^{a}$ Hai-Long He ${ }^{b}$ Shun-Qing Wang ${ }^{c}$ Jing-Yan Tang ${ }^{d}$ Bing Han ${ }^{e}$ \\ Dong-Hua Zhang $^{f}$ Li-Qiang Wu ${ }^{g}$ De-Pei Wu ${ }^{\text {h }}$ Wei Li ${ }^{i} \quad$ Ling-Hui Xia $^{j}$ \\ Huan-Ling Zhu ${ }^{k}$ Feng Liu' Hong-Xia Shi ${ }^{m}$ Xi Zhang $^{\text {n }}$ Fang Zhou $^{\circ}$ \\ Jian-Da Hu ${ }^{p}$ Jian-Pei Fang ${ }^{q}$ Xie-Qun Chen ${ }^{r}$ Tie-Zhen Ye ${ }^{s}$ Ying-Min Liang $^{t}$ \\ Jie Jin ${ }^{\mathrm{u}}$ Feng-Kui Zhang ${ }^{\mathrm{V}}$
}

${ }^{a}$ Center for Pediatric Blood Disease, State Key Laboratory of Experimental Hematology, Institute of Hematology and Blood Diseases Hospital, Chinese Academy of Medical Sciences and Peking Union Medical College, Tianjin, China; ${ }^{b}$ Department of Hematology and Oncology, Soochow University Affiliated Children's Hospital, Suzhou, China; 'Department of Hematology, Guangzhou First People's Hospital, Guangzhou Medical University, Guangzhou, China; ${ }^{\mathrm{d}}$ Department of Hematology and Oncology, Shanghai Children's Medical Center Affiliated to Shanghai Jiaotong University School of Medicine, Shanghai, China; ${ }^{\mathrm{e} D e p a r t m e n t ~ o f ~ H e m a t o l o g y, ~ P e k i n g ~ U n i o n ~ M e d i c a l ~}$ College Hospital, Chinese Academy of Medical Sciences, Beijing, China; ${ }^{\mathrm{D}}$ Department of Hematology, Tongji Hospital, Tongji Medical College Huazhong University of Science and Technology, Wuhan, China; ${ }^{9}$ Department of Hematology, Zhejiang Provincial Hospital of Traditional Chinese Medicine, Hangzhou, China; ${ }^{\mathrm{h}}$ Department of Hematology, The First Affiliated Hospital of Soochow University, Suzhou, China; iDepartment of Hematology, The First Hospital of Jilin University, Changchun, China; 'Department of Hematology, Union Hospital, Tongji Medical College, Huazhong University of Science and Technology, Wuhan, China; ${ }^{2}$ Department of Hematology, West China Hospital, Sichuan University, Chengdu, China; 'Department of Hematology, Xiyuan Hospital, Chinese Academy of Chinese Medicine Sciences, Beijing, China; mPeking University People's Hospital, Peking University Institute of Hematology, Beijing Key Laboratory of Hematopoietic Stem Cell Transplantation, Beijing, China; ${ }^{\mathrm{n} D e p a r t m e n t ~ o f ~}$ Hematology, Xinqiao Hospital, Third Military Medical University, Chongqing, China; ${ }^{\circ}$ Department of Hematology, Jinan Military General Hospital, Jinan, China; ${ }^{\text {PD }}$ epartment of Hematology, Fujian Institute of Hematology, Fujian Provincial Key Laboratory on Hematology, Fujian Medical University Union Hospital, Fuzhou, China; ${ }^{9}$ Department of Pediatric Hematology, Sun Yat-Sen Memorial Hospital, Sun Yat-Sen University, Guangzhou, China; ' Department of Hematology, Xijing Hospital, Fourth Military Medical University, Xi'an, China; ${ }^{5}$ Department of Pediatric Hematology, Guangzhou Women and Children's Medical Center, Guangzhou, China; ${ }^{\mathrm{t} D e p a r t m e n t}$ of Hematology, Tangdu Hospital, Fourth Military Medical University, Xi'an, China; "Department of Hematology, The First Affiliated Hospital, School of Medicine, Zhejiang University, Hangzhou, China; ' Department of Anemia Therapeutic Centre, Institute of Hematology and Blood Diseases Hospital, Chinese Academy of Medical Science and Peking Union Medical College, Tianjin, China

\section{Keywords}

Aplastic anemia $\cdot$ China $\cdot$ Treatment $\cdot$ Registry

X.-F.Z., H.-L.H., and S.-Q.W. contributed equally to this work.

\section{Abstract}

Aplastic anemia (AA) is a hematologic disease characterized by pancytopenia and hypocellular bone marrow, potentially leading to chronic anemia, hemorrhage, and infection. The China Aplastic Anemia Committee and British Committee for

\section{KARGER}

E-Mail karger@karger.com www.karger.com/aha
Feng-Kui Zhang

Department of Anemia Therapeutic Centre, Institute of Hematology and Blood Diseases Hospital, Chinese Academy of Medical Science and Peking Union Medical College, 288 Nanjing Road, Tianjin 300020 (China) E-Mail zhfk@ hotmail.com 
Standards in Haematology guidelines recommend hematopoietic stem-cell transplantation (HSCT) or immunosuppressive therapy (IST) comprising antithymocyte globulin (ATG) with cyclosporine (CsA) as initial treatment for AA patients. With limited epidemiological data on the clinical management of AA in Asia, a prospective cohort registry study involving $22 \mathrm{AA}$ treatment centers in China was conducted to describe the disease characteristics of newly diagnosed AA patients and investigate real-world treatment patterns and patient outcomes. Of 340 AA patients, $72.9,12.6$, and 3.5\% were receiving IST, traditional Chinese medicine, and $\mathrm{HSCT}$, respectively, at baseline; only $22.2 \%$ of IST-treated patients received guideline-recommended ATG with CsA initially. Almost all patients received supportive care $(95.6 \%)$ as blood transfusion (97.8\%), antibiotics (63.7\%), and/or hematopoietic growth factors (58.2\%). Overall, $64.8 \%$ achieved a partial or complete response, and $0.9 \%$ experienced relapse. No new safety concerns were identified; serious adverse events were largely unrelated to the treatment regimen. These results demonstrate the need to identify and minimize treatment barriers to standardize and align AA management in China with treatment guideline recommendations and further improve patient outcomes. @2019 The Author(s)

Published by S. Karger AG, Basel

\section{Introduction}

Aplastic anemia (AA) is characterized by pancytopenia and hypocellular bone marrow and can result in serious clinical manifestations such as chronic anemia, hemorrhage, and infection [1]. While the majority of acquired AA cases are considered idiopathic, AA has also been linked to environmental or occupational toxins such as benzene, viral infection, contaminated water sources, and exposure to animal fertilizers and agricultural pesticides $[2,3]$.

The estimated annual incidence of AA in Western countries is $1.5-2.3$ per million inhabitants [4-6], while in Asia it is 2-3 times higher (3.0-5.0 per million) [3, 7-9]. Previous epidemiological studies have found the incidence of AA in China to be 7.4 per million (1.4 per million with severe AA [SAA] and 6.0 per million with nonsevere AA [NSAA]) $[10,11]$.

The aims of AA therapy are to manage symptoms related to anemia and thrombocytopenia, as well as to prevent serious infection and hemorrhage, with transfusion and other supportive care measures, and to restore hematopoiesis with hematopoietic stem-cell transplantation (HSCT) or immunosuppressive therapy (IST) [12].
The British Committee for Standards in Haematology (BCSH) recommends upfront HSCT for young and adult patients with SAA who have a matched-sibling donor (MSD), with careful comorbidity assessment in those aged 35-50 years. Unrelated-donor HSCT in adults can be considered after lack of response to one course of IST [12]. For patients not suitable for HSCT, IST with horse antithymocyte globulin (ATG) combined with cyclosporine (CsA) is recommended [12]. In 2010, the China Aplastic Anemia Committee published the China Aplastic Anemia Consensus, which largely echoes the recommendations from the BCSH. IST (ATG or antilymphocyte globulin [ALG] in combination with CsA is preferred) is recommended for use in patients with severe disease who are $\geq 40$ or $<40$ years old without an MSD [13, 14]. While the BCSH guidelines recommend horse ATG, in combination with CsA, as the firstline IST regimen [12], horse ATG remains unavailable in many countries worldwide. Rabbit ATG is used widely in Asia, with survival outcomes demonstrated to be comparable to those for horse ATG [15]. Indeed, the China Aplastic Anemia Committee recommends rabbit ATG as a first-line IST regimen [13, 14]. Supportive care, including blood transfusions, iron chelation therapy, and anti-infective medications, is recommended to prevent anemia, infection, and uncontrolled bleeding [12-14].

Epidemiological data around treatment patterns in Asia are limited. However, a large single-center study conducted in northern India showed that only 1 in 5 patients was treated in accordance with guideline recommendations and received either HSCT or IST. Approximately $30 \%$ of patients either did not receive any treatment or were managed with androgen monotherapy, while approximately half received CsA alone or in combination with stanozolol [16].

While it has been reported that patients with AA in China are managed with a wide variety of treatment options, including ATG/ALG + CsA, CsA + androgen, CsA or androgen alone, supportive care alone, and traditional Chinese medicine (TCM) [14], national epidemiological data remain unavailable. This prospective cohort registry study was thus conducted to systematically describe the disease characteristics of newly diagnosed AA patients in China and investigate real-world treatment patterns and outcomes there. This study also aimed to identify differences between real-world clinical practice and guideline recommendations in order to address any gaps in provision of care and optimize patient outcomes. 


\section{Methods}

\section{Study Design}

This was a prospective cohort registry study that used data provided by 22 participating sites across China. Newly diagnosed patients presenting between October 31, 2012 and April 23, 2014 were followed for at least 1 year.

\section{Participants}

Site selection was designed to include a wide geographic distribution across regions and areas in China to reflect the different provinces in the country. The selection criteria included physician's interest, experience, compliance with protocol, and availability of resources. Pediatricians and physicians from TCM hospitals were included as a proportion of AA patients in China are treated by these specialists.

Patients had SAA, very severe AA (VSAA), or transfusion-dependent NSAA (TD-NSAA) were diagnosed within 3 months prior to study enrollment according to the modified Camitta criteria $[17,18]$ as follows: SAA, bone marrow cellularity of $<25 \%$ (or $25-$ $50 \%$ with $<30 \%$ residual hematopoietic cells) plus at least two of the following: (i) neutrophil count $<0.5 \times 10^{9} / \mathrm{L}$; (ii) platelet count $<20 \times 10^{9} / \mathrm{L}$, and (iii) reticulocyte count $<20 \times 10^{9} / \mathrm{L}$; VSAA, meeting the criteria of SAA, with neutrophil count $<0.2 \times 10^{9} / \mathrm{L}$; TDNSAA, AA not fulfilling the criteria for SAA or VSAA, but with at least two of the following: (i) hemoglobin $<100 \mathrm{~g} / \mathrm{L}$; (ii) platelet count $<50 \times 10^{9} / \mathrm{L}$, and (iii) neutrophil count $<1.5 \times 10^{9} / \mathrm{L}$ and a hypocellular bone marrow, and any of the following indications for transfusion dependence: (i) absolute neutrophil count $<0.5 \times$ $10^{9} / \mathrm{L}$; (ii) platelet count $<20 \times 10^{9} / \mathrm{L}$, or (iii) hemoglobin $<80 \mathrm{~g} / \mathrm{L}$ and reticulocyte count $<20 \times 10^{9} / \mathrm{L}$. Treatment with IST regimens for $\leq 4$ weeks, supportive care measures, or TCM prior to enrollment were allowed. Patients with congenital AA, myelodysplastic syndrome, classic paroxysmal nocturnal hemoglobinuria, and AA attributed to treatment for malignancy were excluded.

\section{Data Collection}

Patient data were collected by investigators every 3 months in the first year and every 6 months from the second year until the end of the study (when the last enrolled patient had been followed up for 1 year). Data were collected using paper case report forms from hospital case notes. After collection, data were entered into a computer system by the sponsor, and any requests for additional data were submitted to the investigator. The information collected included demographic data and disease characteristics, patterns of any prior treatments, type and duration of on-study medications, response to treatment, reasons for switching or discontinuing treatment, and safety outcomes.

Treatment types were classified as HSCT, IST-containing treatment (ATG + CsA, CsA + androgen, CsA alone, and others), TCM, androgen monotherapy, supportive care measures (transfusion, hematopoietic growth factors, protective isolation, antibiotics, and iron chelation), and other (those not included as HSCT, IST, TCM, supportive care, or androgen monotherapy).

Response to treatment was evaluated at each study visit. For each treatment, the most recent evaluation of response was included in this analysis. If a patient switched to another treatment, the efficacy evaluation was restarted to assess the efficacy of the new treatment. Response was evaluated in accordance with BCSH guidelines [12]. At the end of the follow-up period, treatment out- comes were assessed as partial response (SAA/VSAA: transfusion independent and no longer meeting the criteria for severe disease; TD-NSAA: transfusion independent, doubling or normalization of at least one cell line, or increase of baseline hemoglobin $>30 \mathrm{~g} / \mathrm{L}$ [if initially $<60$ ], neutrophil count $>1.5 \times 10^{9} / \mathrm{L}$ [if initially $<0.5$ ] or platelet count $>20 \times 10^{9} / \mathrm{L}$ [if initially $<20$ ]), complete response (SAA/VSAA/TD-HSAA: normal hemoglobin concentration, neutrophil count $>1.5 \times 10^{9} / \mathrm{L}$, and platelet count $>150 \times 10^{9} / \mathrm{L}$ ), no response (SAA/VSAA: fulfilling severe disease criteria; TD-NSAA: worse or not meeting any criteria for partial or complete response), and relapse (SAA/VSAA/TD-HSAA: requiring further transfusion or any treatment after maintaining response for 3 months). For SAA and VSAA, treatment outcomes were assessed as partial response (transfusion dependent and no longer meeting criteria for severe disease), complete response (normal hemoglobin concentration, neutrophil count $>1.5 \times 10^{9} / \mathrm{L}$ and platelet count $>150$ $\times 10^{9} / \mathrm{L}$ ), no response (fulfilling severe disease criteria), and relapse (requiring further transfusion or any treatment after maintaining response for 3 months) at the end of the follow-up period. For TDNSAA, treatment outcomes were assessed as partial response (transfusion independent, doubling or normalization of at least one cell line, or increase of baseline hemoglobin $>30 \mathrm{~g} / \mathrm{L}$ [if initially $<60$ ], neutrophil count $>1.5 \times 10^{9} / \mathrm{L}$ [if initially $<0.5$ ], or platelet count $>20 \times 10^{9} / \mathrm{L}$ [if initially $\left.<20\right]$ ), complete response (normal hemoglobin concentration, neutrophil count $>1.5 \times 10^{9} / \mathrm{L}$ and platelet count $>150 \times 10^{9} / \mathrm{L}$ ), no response (worse or not meeting any criteria for partial or complete response), and relapse (requiring further transfusion or any treatment after maintaining response for 3 months) at the end of the follow-up period. Adverse events related to treatment were recorded and coded using the Medical Dictionary for Regulatory Activities version 15.0.

\section{Statistical Analysis}

All patients who provided informed consent, had a documented diagnosis of SAA, VSAA, or TD-NSAA, and either were treatment naïve or had received treatment for less than 2 months prior to enrollment were included in the full analysis set (FAS). The safety population comprised all enrolled patients who had safety data from at least one study visit. The planned sample size for analysis was 350 and was based on a 95\% confidence interval for determining treatment pattern proportions of at least $25 \%$.

Continuous variables are expressed as median values with ranges, and categorical variables are summarized using descriptive statistics. The $\chi^{2}$ test was used to compare categorical variables across subgroups of patients. For all tests, a two-sided $p$ value $\leq 0.05$ was considered statistically significant. There was no imputation of missing data for any of the analyses. Statistical analyses were conducted using $S A S^{\circledR}$ version 9.4 or later.

\section{Results}

\section{Patient Demographics and Baseline Characteristics}

A total of 352 patients were enrolled from 22 sites in this study between October 31, 2012 and April 23, 2014. Most patients were recruited from participating sites in northern $(34.0 \% ; n=121 / 352)$ and eastern regions of China $(30.0 \% ; n=106 / 352$; Fig. 1$)$. 


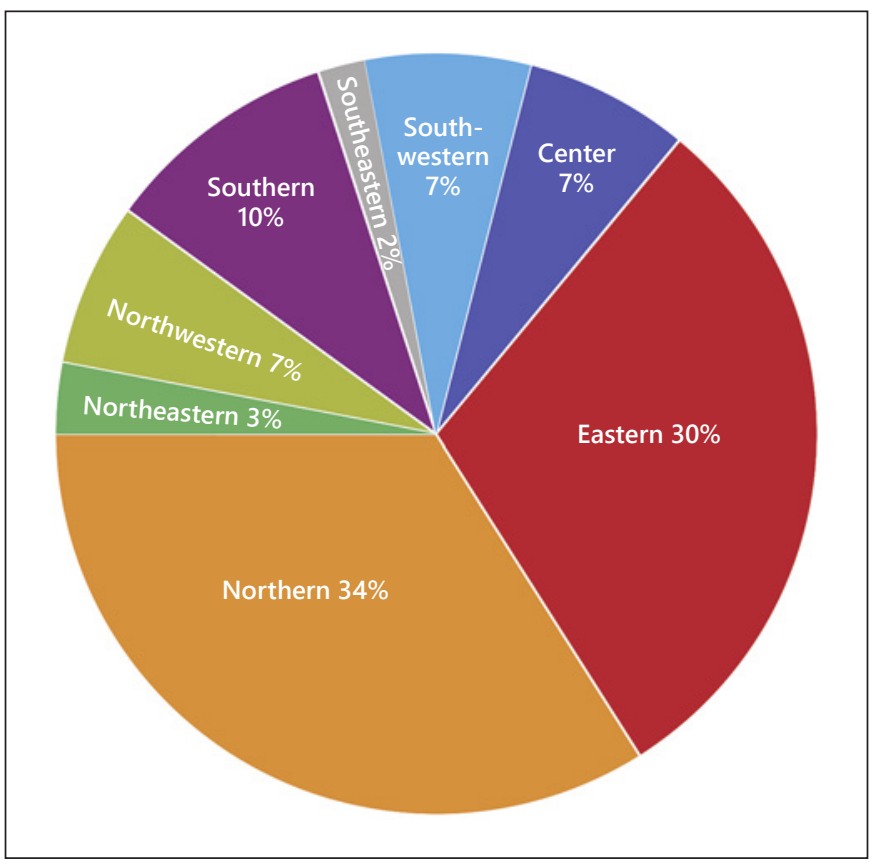

Fig. 1. Distribution of AA patients enrolled in the registry in China.

Patients were followed up for a mean of $485 \pm 213$ days. The median age was 21 years (range $0-84)$, and $65.0 \%(n=$ 229) of patients were aged 14 years or above (Table 1 ).

Of the 352 patients enrolled, the majority $(61.9 \% ; n=$ $218)$ were diagnosed as having SAA, while $23.6 \%(n=83)$ had VSAA and $14.5 \%(n=51)$ had TD-NSAA. The majority of patients $(54.8 \% ; n=193)$ had an Eastern Cooperative Oncology Group (ECOG) score of 1 at the time of enrollment. Most patients presented at the time of enrollment with anemia (92.9\%), hemorrhage (72.2\%), and infection (56.8\%). The mean hemoglobin count was $76.07 \mathrm{~g} / \mathrm{L}$ (SD $16.97 \mathrm{~g} / \mathrm{L})$, with a median platelet count of $24.00 \times 10^{9} / \mathrm{L}$ (range 1.0-263.0), median absolute neutrophil count of $0.5260 \times 10^{9} / \mathrm{L}$ (range $0.000-15.990$ ), and median absolute reticulocyte count of $0.0122500 \times 10^{9} / \mathrm{L}$ (range 0.000 $0.168)$. A total of 245 cases (69.6\%) received cytogenetic testing at baseline; 230 (93.9\%) cases showed normal cytogenetics, 5 had a normal karyotype but poor cell proliferation, 5 were not available for chromosome analysis, and chromosomal abnormalities were found in 5 cases.

\section{Treatment Pattern}

Initial AA Treatment

Among the 352 enrolled patients, 12 either had no clinical documentation of an AA diagnosis or had received IST treatment for more than 8 weeks and were excluded from the FAS analyses $(n=340)$. At baseline, $248(72.9 \%)$ of the 340 patients included in the FAS received IST, 12 (3.5\%) received HSCT, 43 (12.6\%) received TCM, and only 8 (2.4\%) used androgen alone (Table 2 ). Of the 43 patients who received TCM, only 3 used TCM as the main treatment regimen for AA. Almost all (95.6\%) newly diagnosed AA patients received supportive care at baseline.

\section{HSCT}

At baseline, 12 patients (7 SAA, 3.3\%, and 5 VSAA, $6.0 \%)$ received HSCT as the initial choice of treatment (Table 2). In the SAA group, 2 received only bone marrow transplantation, 1 received only peripheral blood stem cell transplantation, 3 received both from related donors, while 1 received cord blood transplantation. In the VSAA group, 1 received only bone marrow transplantation, 3 received bone marrow transplantation as well as peripheral blood stem cell transplantation from related donors, while 1 received haploid transplantation. Over the course of the study, 52 patients were switched to HSCT: 37 in the first 3 months, 4 in months $3-6,3$ in months $6-9,1$ in months $9-12$, and 3 between month 12 and the end of follow-up. By the end of the follow-up period, 60 patients had received HSCT (Table 3 ).

\section{IST-Containing Regimens}

Of the 248 patients who received IST-containing regimens at baseline (Fig. 2), the majority were treated with CsA either alone $(n=86)$ or in combination with androgen $(n=76)$. Overall, a total of 84 patients received ATGcontaining therapy with rabbit ATG (Thymoglobulin ${ }^{\circledR}$, $76.2 \%$, mean daily dose $135.8 \mathrm{mg}$ ), ATG-Fresenius (2.4\%, mean daily dose $166.7 \mathrm{mg}$ ), or porcine ALG (21.4\%, mean daily dose 1,311.4 mg). Only 1 in 5 patients received ATG in combination with CsA as the initial AA treatment, as recommended by the guidelines.

Trends of IST use as initial AA treatment were largely similar between SAA and VSAA patients. However, almost all TD-NSAA patients received either CsA alone or in combination with androgen, and only 1 patient received the guideline-recommended ATG in combination with CsA.

\section{Supportive Care Measures}

Almost all patients received supportive care $(n=325 / 340$; 95.6\%). At baseline, the most common supportive care measure received by patients was blood transfusion (97.8\%), followed by antibiotics (63.7\%) and hematopoietic growth factors (58.2\%). Among the 318 patients who received blood transfusions, 225 (70.8\%) used leukocyte-reduced blood and $89(28.0 \%)$ used irradiated blood (Table 4). 
Table 1. Patient demographics and baseline characteristics

\begin{tabular}{lcccc}
\hline Characteristic & SAA $(n=218)$ & VSAA $(n=83)$ & TD-NSAA $(n=51)$ & Total $(n=352)$ \\
\hline Male & $110(50.5)$ & $42(50.6)$ & $30(58.8)$ & $182(51.7)$ \\
Median age, years & $21.0(0-84)$ & $21.0(3-79)$ & $23.0(4-68)$ & $21.0(0-84)$ \\
ECOG score & & & \\
0 & $37(17.0)$ & $10(12.0)$ & $12(23.5)$ & $59(16.8)$ \\
1 & $130(59.6)$ & $34(41.0)$ & $29(56.9)$ & $193(54.8)$ \\
2 & $37(17.0)$ & $34(41.0)$ & $9(17.6)$ & $14(4.0)$ \\
3 & $10(4.6)$ & $4(4.8)$ & 0 & $1(0.3)$ \\
4 & $1(0.5)$ & 0 & 0 & $5(1.4)$ \\
5 & 0 & 0 & $1(2.0)$ & $200(56.8)$ \\
Unknown & $3(1.4)$ & $1(1.2)$ & $12(23.5)$ & $327(92.9)$ \\
Presenting symptoms ${ }^{1}$ & $128(58.7)$ & $60(72.3)$ & $43(84.3)$ & $254(72.2)$ \\
Infection & $207(95.0)$ & $77(92.8)$ & $27(52.9)$ & $12(3.4)$ \\
Anemia & $161(73.9)$ & $66(79.5)$ & 0 & $230 / 245(93.9)$ \\
Hemorrhage & $11(5.0)$ & $1(1.2)$ & & \\
Other symptoms & $147 / 157(93.6)$ & $55 / 58(94.8)$ & & \\
Normal cytogenetics & & & \\
\hline
\end{tabular}

Overall Treatment Types Received by AA Patients

Over the course of the study, there were 131 occurrences of treatment switching. Most patients $(n=86 / 131$; $66.0 \%)$ switched from an initial treatment to a different therapy in the first 3 months of the study, mostly because of unsatisfactory treatment efficacy. Throughout the follow-up period, 52 patients switched to HSCT and 49 patients switched to an IST-containing regimen. Sixty patients switched from a non-ATG-containing (including IST and non-IST) regimen to ATG + CsA within the first 3 months. The most commonly used AA treatments over the course of the study were IST-containing regimens and supportive care measures.

\section{Treatment Outcomes}

For each treatment, the most recent evaluation of response was used. In cases where patients switched treatments, the response included in this analysis was that for the treatment they received at the end of follow-up.

In the overall population $(n=332)$, the majority of patients responded to AA treatment: $47.6 \%(n=158)$ of patients achieved a partial response and $17.2 \%(n=57)$ of patients achieved a complete response; $34.4 \%(n=114)$ of patients did not respond to treatment (Fig. 3). Treatment with HSCT was successful (complete or partial response) in $89.4 \%$ of patients, and only 1 patient who had SAA experienced a relapse (Fig. 3). Among all AA patients, com-
Table 2. AA treatment at baseline (FAS)

\begin{tabular}{lcccr}
\hline Treatment & $\begin{array}{l}\text { SAA } \\
(n=209)\end{array}$ & $\begin{array}{l}\text { VSAA } \\
(n=83)\end{array}$ & $\begin{array}{l}\text { TD-NSAA } \\
(n=48)\end{array}$ & $\begin{array}{l}\text { Total } \\
(n=340)\end{array}$ \\
\hline HSCT & $7(3.3)$ & $5(6.0)$ & 0 & $12(3.5)$ \\
IST & $155(74.2)$ & $58(69.9)$ & $35(72.9)$ & $248(72.9)$ \\
TCM & $32(15.3)$ & $9(10.8)$ & $2(4.2)$ & $43(12.6)$ \\
$\begin{array}{l}\text { Supportive care } \\
\text { Androgen }\end{array}$ & $204(97.6)$ & $83(100)$ & $38(79.2)$ & $325(95.6)$ \\
$\quad$ monotherapy & $7(3.3)$ & 0 & $1(2.1)$ & $8(2.4)$ \\
\hline
\end{tabular}

Data are presented as $n(\%)$. Patients may have received more than 1 treatment. AA, anaplastic anemia; FAS, full analysis set; SAA, severe AA; VSAA, very severe AA; TD-NSAA, transfusion-dependent non-severe AA; HSCT, hematopoietic stem-cell transplantation; IST, immunosuppressive therapy; TCM, traditional Chinese medicine.

plete response was only achieved with HSCT and IST treatment. Nevertheless, only $0.9 \%$ (2 IST and 1 HSCT) of patients experienced a relapse (Fig. 3).

Among patients treated with IST-containing regimens, $62.8 \%$ achieved either a complete or a partial response. The response rate was highest among patients who were treated with CsA in combination with androgen (overall response $56.3 \%, n=67$; complete response $14.3 \%, n=17$; partial response $42.0 \%, n=50$ ), of whom 
Fig. 2. Use of IST regimens as initial AA treatment options by disease severity.
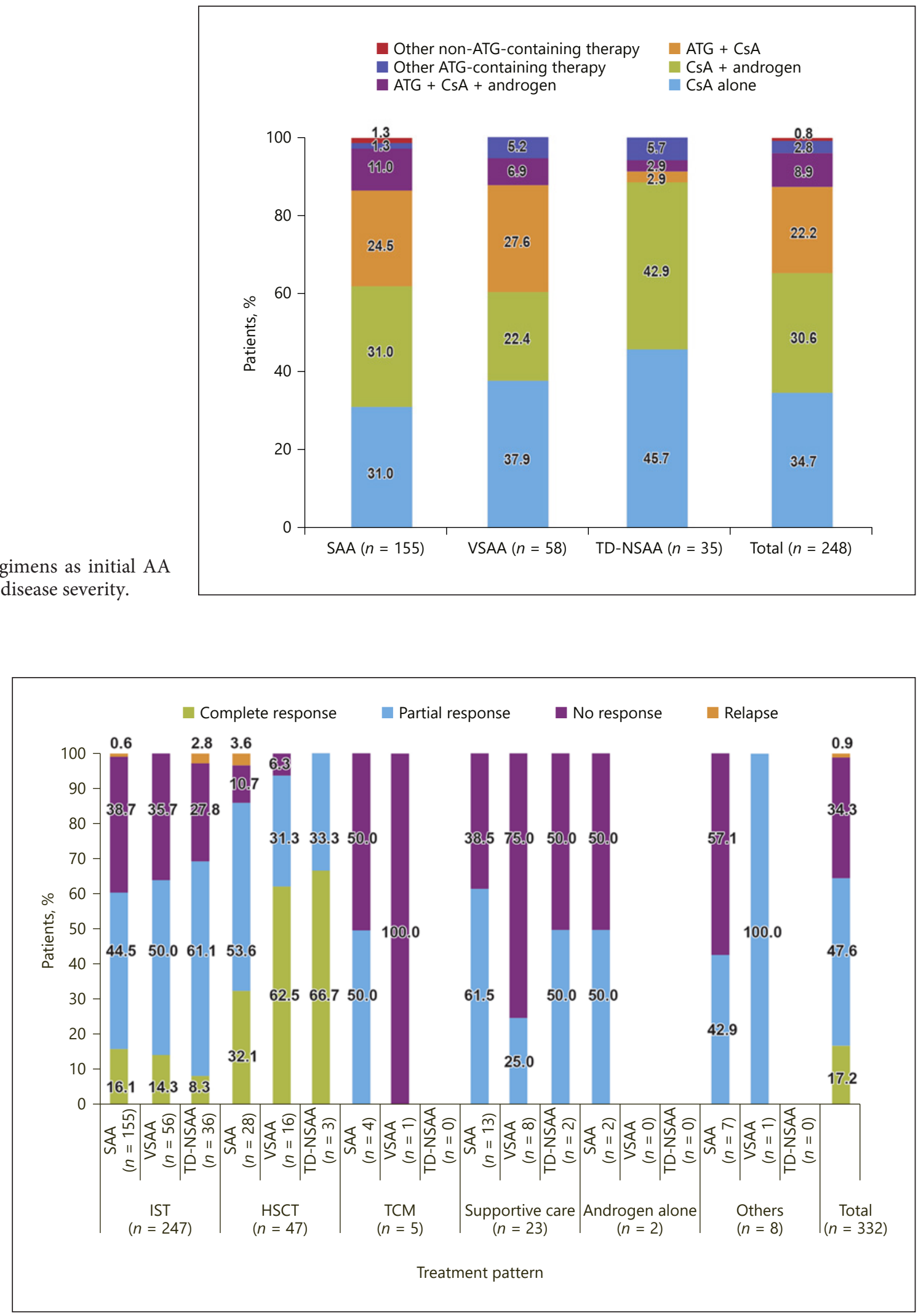

Fig. 3. Outcomes to AA treatment received at the end of the follow-up period. 
Table 3. Overall treatments received by AA patients throughout the study period (from baseline to end of followup)

\begin{tabular}{lclcc}
\hline Regimen & SAA $(n=209)$ & VSAA $(n=83)$ & TD-NSAA $(n=48)$ & Total $(n=340)$ \\
\hline HSCT containing & $38(18.2)$ & $18(21.7)$ & $4(8.3)$ & $60(17.6)$ \\
IST containing & $186(89.0)$ & $72(86.7)$ & $45(93.8)$ & $303(89.1)$ \\
TCM containing & $56(26.8)$ & $16(19.3)$ & $7(14.6)$ & $79(23.2)$ \\
Supportive care containing & $207(99.0)$ & $83(100.0)$ & $39(81.3)$ & $329(96.8)$ \\
Androgen monotherapy containing & $8(3.8)$ & 0 & $1(2.1)$ & $9(2.6)$ \\
Others & $15(7.2)$ & $8(9.6)$ & $2(4.2)$ & $25(7.4)$ \\
\hline
\end{tabular}

Data are presented as $n(\%)$. Patients may have received more than 1 treatment. AA, anaplastic anemia; SAA, severe AA; VSAA, very severe AA; TD-NSAA, transfusion-dependent non-severe AA; HSCT, hematopoietic stem-cell transplantation; IST, immunosuppressive therapy; TCM, traditional Chinese medicine.

Table 4. Use of supportive care measures as initial AA treatment by disease severity

\begin{tabular}{lcccc}
\hline Supportive care measure & SAA $(n=209)$ & VSAA $(n=83)$ & TD-NSAA $(n=48)$ & Total $(n=340)$ \\
\hline Any supportive care & $204(97.6)$ & $83(100)$ & $38(79.1)$ & $325(95.6)$ \\
Blood transfusion & $199(97.5)$ & $83(100)$ & $36(94.7)$ & $318(97.8)$ \\
Hematopoietic growth factors & $122(59.8)$ & $57(68.7)$ & $10(26.3)$ & $189(58.2)$ \\
Protective isolation & $97(47.5)$ & $51(61.4)$ & $4(10.5)$ & $152(46.8)$ \\
Antibiotics & $123(60.3)$ & $71(85.5)$ & $13(34.2)$ & $207(63.7)$ \\
Iron chelation & 0 & $1(1.2)$ & 0 & $1(0.3)$ \\
Other & $7(3.4)$ & 0 & $2(5.3)$ & $9(2.8)$ \\
\hline
\end{tabular}

Data are presented as $n(\%)$. Patients may have received more than 1 type of supportive care measure. AA, anaplastic anemia; SAA, severe AA; VSAA, very severe AA; TD-NSAA, transfusion-dependent non-severe AA.

$92.0 \%$ had severe or very severe disease, followed by CsA alone (overall response $53.8 \%, n=86$; complete response $10.0 \%, n=16$; partial response $43.8 \%, n=70$ ). Of the 180 patients treated with ATG in combination with CsA by the end of follow-up, $96.0 \%$ of whom had severe or very severe disease, 9.4\% $(n=17)$ achieved a complete response and $40.0 \%(n=72)$ achieved a partial response.

\section{Safety}

Of the 352 patients included in the safety analysis set, a total of 51 serious adverse events (SAEs) were reported by 35 patients $(9.9 \%)$. The most common SAEs were infections (3.1\%), nervous system disorders (2.3\%), and blood and lymphatic system disorders (1.7\%). None of the patients in the TD-NSAA group experienced any SAE, except for 1 patient who had a decreased blood platelet count. For 2 patients in the SAA group, the SAEs were judged to be related to the treatment received during the study, and for 6 patients (3 SAA and 3 VSAA), SAEs were possibly related to the treatment. The final outcome was that 30 out of the 35 patients (18 SAA and 12 VSAA) died; in the remaining 5 patients, SAEs were resolved or recovered from.

\section{Discussion}

To the best of our knowledge, this is the first prospective cohort registry study evaluating disease characteristics, treatment practices, and outcomes of newly diagnosed AA patients in China. Our registry study revealed that most AA patients in China received treatment and care from institutions in northern and eastern regions of the country, where most main or large AA treatment centers are located. Consistent with other reports from China and findings from other countries in Asia, the majority of AA patients in this registry had severe disease and presented at a median age of 23 years $[16,19,20]$.

Findings from this registry study showed that the rate of first-line HSCT in this study was low; only $3.5 \%$ of newly 
diagnosed AA patients received HSCT as the initial AA treatment. In comparison, $5-8 \%$ of patients with acquired AA in India received transplantation as first-line treatment $[16,21]$. The low rate in China may be related to the shortage of sibling donors, the high cost of therapy, and the lack of resources and infrastructure to support HSCT for the large population of patients. Furthermore, a survey of treatment practices for pediatric AA in North America found that almost $40 \%$ of physicians considered IST as the first-line treatment in HSCT candidates for various reasons, including family concerns regarding fertility, anticipated delay of treatment, and psychosocial barriers associated with transplantation [22]. However, our registry study did not record reasons for patients not receiving HSCT.

The majority of AA patients in China receive IST as initial treatment either as CsA alone or in combination with androgen. However, of the patients who received IST in our study, only 1 in 5 received the guideline-recommended IST regimen of ATG in combination with CsA as initial treatment. It is known that institutions in North America adhere to recommendations made by major AA treatment guidelines and choose the combination of ATG and CsA as the preferred IST regimen [22]. In China, however, androgen is prescribed more widely than ATG as a combination partner for CsA as it is cheaper than ATG. Indeed, erythropoiesis stimulants such as androgen are recommended by the Chinese consensus statement for use with IST. This finding is consistent with treatment practices in India, where CsA alone or in combination with androgen is the most common treatment regimen owing to the high cost of HSCT and ATG [16].

Nearly all patients in our registry cohort received supportive care, mainly in the form of antibiotics and blood transfusions. Only 1 patient who had VSAA received iron chelation therapy, potentially owing to the high cost of chelators. Approximately half of all patients, most of whom had very severe disease and were at high risk for infection, were cared for in protective isolation during hospitalization to reduce the risk for infections, in line with $\mathrm{BCSH}$ guideline recommendations. Although 12.6\% of patients received TCM, this was not the main AA treatment for nearly all of those patients, and most Chinese physicians regard TCM as a complementary or supportive measure in AA management. Therefore, a preference for use of TCM is unlikely to interfere with adherence to treatment guidelines in China.

In China, $65 \%$ of newly diagnosed AA patients, most of whom received IST, achieved either a partial or complete response with current treatment patterns. Up to $90 \%$ of patients achieved a partial or complete response

AA Prospective Cohort Registry Study in China with HSCT, and only 1 patient experienced a relapse. AA patients in China whose first-line HSCT has failed are typically treated with non-ATG-containing ISTs as the second-line treatment option owing to cost barriers.

In our registry cohort, half of all patients treated with rabbit ATG in combination with CsA achieved a partial or complete response, which is comparable with findings from clinical studies in Asia that have demonstrated overall response rates of $58-70 \%[15,16,21]$. Findings from our real-world evaluation showed that more patients who were treated with CsA alone or in combination with androgen achieved a partial or complete response than those treated with a combination of ATG and CsA. This may be a result of the higher proportion of SAA and VSAA patients in the ATG-containing regimen group compared with the non-ATG-containing regimen group. Furthermore, the efficacy of treatments may have been impacted by the delay or interval between diagnosis and initiation of ATG [12-14]. Despite being recommended as the first-line IST, ATG in combination with CsA may pose financial barriers to the treatment of AA in China. As such, data from our real-world study suggest that CsA alone or in combination with androgen is an acceptable, safe, effective, and affordable alternative that offers comparable outcomes for AA patients in China. No safety concern has been identified in this registry study. The majority of SAEs were not related to the treatment.

This registry study is a real-world analysis and is associated with several challenges. There was a high rate of loss to follow-up (156 of the 205 non-completers in the study), which may have had an impact on the outcomes assessment; however, this was relatively even between disease severity groups. It is possible that the distribution of patients who did not complete the study may have been unequal between treatment groups, introducing a source of bias. Patients were followed up for only slightly over a year, which may be too short to detect any differences in failurefree survival between the different IST-containing regimens. The short follow-up period and the high rate of patients lost to follow-up made the analysis of long-term outcomes, including efficacy parameters, difficult. This study does not address diagnosis practices for AA in China; as such, the time between diagnosis and treatment initiation is unknown. The treatment period was indefinite, due to huge variations in treatment duration between centers. Finally, there is great diversity in populations, incomes, and standards of care across China. Although this study aimed to capture a representative selection of the main AA treatment centers in China, it likely does not truly represent the spectrum of AA management in the country.

Acta Haematol 2019;142:162-170 


\section{Conclusions}

While the majority of AA patients in China were treated with IST, few received the guideline-recommended combination of ATG and CsA, most likely owing to financial constraints. Nevertheless, the majority of patients treated with any IST achieved a complete or partial response. Barriers to treatment and gaps in provision of care should be identified and minimized so that management of AA patients in China can be standardized and aligned with treatment guideline recommendations to further improve outcomes for patients.

\section{Acknowledgments}

The authors would like to thank the patients and their families for their contribution to this study. This study was sponsored by Sanofi. Writing assistance was provided by Stefanie Chuah from Mudskipper Business Ltd, funded by Sanofi China Pharmaceuticals.

\section{Statement of Ethics}

This registry study was conducted in accordance with the ethical standards of the institutional and/or national research committee, the guidelines for Good Epidemiology Practice, and the principles of the Declaration of Helsinki. The registry protocol was reviewed and approved by a steering committee, and all patients or their parents or legal guardians provided written informed consent.

\section{Disclosure Statement}

The authors declare that they have no conflicts of interest. A summary of relevant information will be published with the report.

\section{References}

1 DeZern AE, Guinan EC. Aplastic anemia in adolescents and young adults. Acta Haematol. 2014;132(3-4):331-9.

2 Young NS, Kaufman DW. The epidemiology of acquired aplastic anemia. Haematologica. 2008 Apr;93(4):489-92.

3 Issaragrisil S, Kaufman DW, Anderson T, Chansung K, Leaverton PE, Shapiro S, et al. The epidemiology of aplastic anemia in Thailand. Blood. 2006 Feb;107(4):1299-307.

4 Mary JY, Baumelou E, Guiguet M; The French Cooperative Group for Epidemiological Study of Aplastic Anemia. Epidemiology of aplastic anemia in France: a prospective multicentric study. Blood. 1990 Apr;75(8):164653.

5 Tweddle DA, Reid MM. Aplastic anemia in the northern region of England. Acta Paediatr. 1996 Nov;85(11):1388-9.

6 Montané E, Ibáñez L, Vidal X, Ballarín E, Puig R, García N, et al.; Catalan Group for Study of Agranulocytosis and Aplastic Anemia. Epidemiology of aplastic anemia: a prospective multicenter study. Haematologica. 2008 Apr; 93(4):518-23.

7 Issaragrisil S, Leaverton PE, Chansung K, Thamprasit T, Porapakham Y, Vannasaeng S, et al.; The Aplastic Anemia Study Group. Regional patterns in the incidence of aplastic anemia in Thailand. Am J Hematol. 1999 Jul; 61(3):164-8.

8 Kojima S. Aplastic anemia in the Orient. Int J Hematol. 2002;76(Suppl 2):173-4.
9 Wang W, Wang XQ, Li P, Lin GW. [Incidence of adult aplastic anemia in Shanghai, China]. Zhonghua Nei Ke Za Zhi. 2011 Apr;50(4): 284-6. Chinese.

10 Shao Z, Yang C, Yang T. [Epidemiology of aplastic anaemia]. Zhonghua Xue Ye Xue Za Zhi. 1988;9:113-5. Chinese.

11 Yang C, Zhang X. Incidence survey of aplastic anemia in China. Chin Med Sci J. 1991 Dec; 6(4):203-7.

12 Killick SB, Bown N, Cavenagh J, Dokal I, Foukaneli T, Hill A, et al.; British Society for Standards in Haematology. Guidelines for the diagnosis and management of adult aplastic anaemia. Br J Haematol. 2016 Jan;172(2): 187-207.

13 Chinese Aplastic Anemia Committee. [Expert consensus on diagnosis and treatment of aplastic anemia in China]. Zhonghua Xue Ye Xue Za Zhi. 2010;31:790-2. Chinese.

14 Zhang FK. [To standardize the treatment regimen so as to improve the therapeutic outcome of aplastic anemia]. Zhonghua Xue Ye Xue Za Zhi. 2008 Nov;29(11):721-2. Chinese.

15 Chuncharunee S, Wong R, Rojnuckarin P, Chang CS, Chang KM, Lu MY, et al. Efficacy of rabbit antithymocyte globulin as first-line treatment of severe aplastic anemia: an Asian multicenter retrospective study. Int J Hematol. 2016 Oct;104(4):454-61.

16 Mahapatra M, Singh PK, Agarwal M, Prabhu M, Mishra P, Seth T, et al. Epidemiology, Clinico-haematological profile and management of aplastic anaemia: AIIMS experience. J Assoc Physicians India. 2015 Mar;63(3 Suppl):30-5.
17 Camitta BM, Rappeport JM, Parkman R, Nathan DG. Selection of patients for bone marrow transplantation in severe aplastic anemia. Blood. 1975 Mar;45(3):355-63.

18 Bacigalupo A, Hows J, Gluckman E, Nissen C, Marsh J, Van Lint MT, et al. Bone marrow transplantation (BMT) versus immunosuppression for the treatment of severe aplastic anaemia (SAA): a report of the EBMT SAA working party. Br J Haematol. 1988 Oct; 70(2): 177-82.

19 Li X, Feng Y, Wang H, Song M, Jin J, Cui Z, et al. Nutritional status survey of aplastic anemia patients - a single center experience in China. Appl Nurs Res. 2016 May;30:142-7.

20 Adil SN, Burney IA, Kakepoto GN, Khurshid M. Epidemiological features of aplastic anaemia in Pakistan. J Pak Med Assoc. 2001 Dec; 51(12):443-5.

21 Ramzan M, Yadav SP, Zafar MS, Dinand V, Sachdeva A. Outcome of pediatric acquired aplastic anemia: a developing world experience. Pediatr Hematol Oncol. 2014 Feb;31(1): 29-38.

22 Williams DA, Bennett C, Bertuch A, Bessler M, Coates T, Corey S, et al. Diagnosis and treatment of pediatric acquired aplastic anemia (AAA): an initial survey of the North American Pediatric Aplastic Anemia Consortium (NAPAAC). Pediatr Blood Cancer. 2014 May;61(5):869-74. 